\title{
Analysis of Fluorescence Quenching of BPBD by Aniline in Toluene
}

\author{
J Thipperudrappa* and S M Hanagodimath ${ }^{\dagger}$
}

\begin{abstract}
Fluorescence quenching of 2-(4'-t-Butylphenyl)-5-(4"biphenylyl)-1,3,4-oxadiazole (BPBD) by aniline in toluene has been carried out at room temperature by steady state and time resolved fluorescence spectroscopy. The SternVolmer plot by steady state method has been found to be non-linear showing a positive deviation, whereas by timeresolved method it is linear. In order to interpret these results we have used the ground state complex and sphere of action static quenching models. Using these models various rate parameters have been determined. Based on these models, with finite sink approximation model, we conclude that positive deviation Stern-Volmer plot is due to the simultaneous presence of dynamic and static quenching processes.
\end{abstract}

Keywords: Fluorescence quenching, stern-volmer plot, BPBD, static quenching, dynamic quenching, finite sink approximation model.

\section{Introduction}

Fluorescence quenching of organic fluorophores in solution by various chemical species like aniline, bromobenzene, carbon tetra chloride, eththyltrithiocarbonate, halide ions, metal ions etc. has

* Department of Physics, BNM Institute of Technology, Bangalore-560 070, India; jtrphy2007@gmail.com.

† Department of Physics, Gulbarga University, Gulbarga-585 106, India; smhmath@rediffmail.com.

Received: November 2012; Reviewed: December 2012 
been studied by several investigators [1-11]. The accessibility of fluorophores to quenchers can be used to determine the location of probes on macromolecules. This leads to the importance of quenching mechanism not only in physical science but also in chemical, biological and medical sciences. The study has been mainly to understand the nature of bimolecular reactions taking place both under steady state and transient conditions. The fluorescence in bimolecular liquid systems is quenched due to several mechanisms such as static and dynamic quenching, excimer and exciplex formation, charge transfer processes etc.

2-(4'-t-Butylphenyl)-5-(4"-biphenylyl)-1，3，4 -oxadiazole (BPBD) finds various applications as a laser dye and as electron transport layer in Polymer Light Emitting Devices (PLEDs) [12]. It also has potential applications in Quantum dots-based light emitting diodes (QLEDs), solar cells and Organic light emitting diode (OLEDs) as commonly used organic [13], wavelength shifter in the form of liquid and solid solutions in Cherenkov counters [14] and as a scintillation fluid for scintillation counting of polyacrylamide gel slices after electrophoresis [15]. All these applications of BPBD are based on its high quantum yield/fluorescence. The quenching of its fluorescence by a quencher leads to the reduction in efficiency of devices which make use BPBD as their counterpart. Hence, fluorescence quenching of BPBD by a chemical species in general, by aniline in particular has practical importance.

In the present study, we have used steady state and time-resolved experimental methods to investigate the quenching of BPBD by aniline in toluene with a view to understand the nature of quenching mechanism.

\section{Materials and methods}

The solute BPBD was obtained from Sigma -Aldrich, USA and is of scintillation grade. The molecular structure of BPBD is shown in Fig. 1. The quencher aniline was analytical grade and used after double distillation. The toluene was of spectroscopic grade and is used without any further purification. The solutions were prepared at very low fixed concentration of BPBD $\left(1 \times 10^{-5} \mathrm{M}\right)$ to avoid self absorption effects and varying the quencher concentration $(0.000 \mathrm{M}$ 
to $0.010 \mathrm{M})$. Fluorescence spectrophotometer F-2000 was used for fluorescence intensity measurements, with perpendicular geometry. BPBD has maximum absorption of $305 \mathrm{~nm}$ in toluene. Therefore, it was excited at $305 \mathrm{~nm}$ and fluorescence spectrum was recorded in the absence and presence of quencher. The experimental values are reproducible within $5 \%$ of the experimental error. The Measurement of fluorescence lifetime of BPBD with and without quencher was made by using single photon9 counting time-domain spectrofluorimeter (Edinburgh Instrument, UK, Model EI-199). Hydrogen was used to fill the lamp of the single photon counting (SPC) system at $0.6 \mathrm{~atm}$ pressure, keeping the electrode separation $0.7 \mathrm{~mm}$, applying the discharge voltage of about $7 \mathrm{kV}$ and pirating the lamp at a frequency $30 \mathrm{kHz}$. With this setting, lamp pulses having pulse width of about $1 \mathrm{~ns}$ at FWHM were obtained.

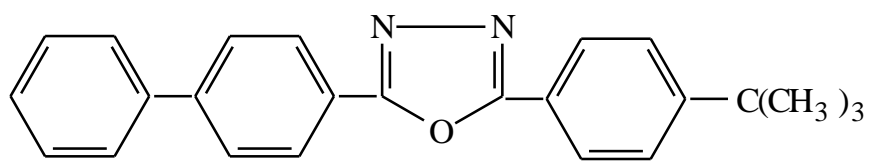

Fig.1. Molecular structure of BPBD

\section{Results and discussion}

The steady state fluorescence intensities $\mathrm{I}_{0}$ and I were measured in the absence and presence of quencher respectively in toluene. The fluorescence spectrum of BPBD in the presence of different concentrations of quencher aniline is shown in Fig. 2. From Fig. 2, it is clear that fluorescence of BPBD is efficiently quenched by the addition of aniline. Further, fluorescence lifetime of BPBD was measured in toluene in the absence and presence of quencher. The fluorescence decay curves of BPBD with and without quencher are double exponential according to

$$
I(t)=a_{1} \exp \left(-t / \tau_{1}\right)+a_{2} \exp \left(-t / \tau_{2}\right)
$$


where the subscripts 1 and 2 refer to the shorter and longer preexponential components respectively of the decay and, $a_{1}$ and $a_{2}$ are the corresponding relative amplitudes. The lifetime values were obtained by fitting experimental decay profiles to double exponential function (using reconvolution procedure) to get $\chi 2$ values very close to unity. The average lifetime $\langle\tau\rangle$ in each case is calculated according to equation $\langle\tau\rangle={ }^{a_{1}} \tau 1+{ }^{a_{2}} \tau 2$. Where $\tau 1$ and $\tau 2$ are the fluorescence lifetimes corresponding to amplitudes $a_{1}$ and ${ }^{a_{2}}$ respectively. The corresponding data is given in Table 1. From Table 1, it can be observed that lifetime of BPBD decreases with increase in quencher concentration.

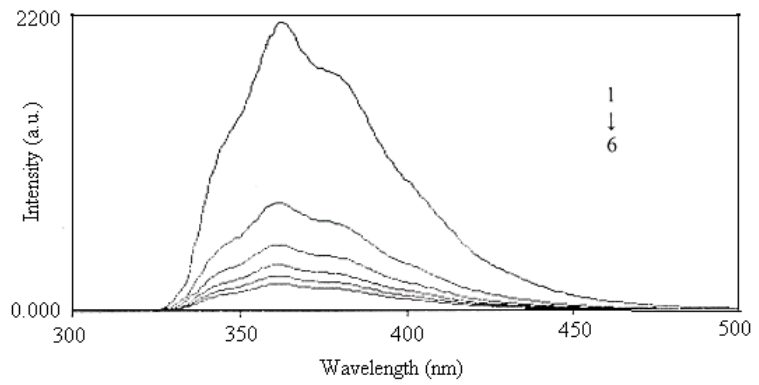

Fig. 2. Fluorescence emission spectra of BPBD in the presence of aniline in toluene at Concentrations of aniline (in M/L) (1) 0.000 (2) 0.002 (3) 0.004 (4) 0.006 (5) 0.008 (6) 0.010.

\begin{tabular}{|c|c|c|c|c|c|}
\hline $\begin{array}{c}\text { Quencher } \\
\text { concentration } \\
(\boldsymbol{Q}) \mathbf{M} / \mathbf{L}\end{array}$ & $\begin{array}{c}\tau_{\boldsymbol{I}} \\
(\mathbf{n s})\end{array}$ & $\boldsymbol{a}_{\boldsymbol{1}}$ & $\begin{array}{c}\boldsymbol{\tau}_{2} \\
(\mathbf{n s})\end{array}$ & $\boldsymbol{a}_{\boldsymbol{2}}$ & $\begin{array}{c}\text { Average } \\
\text { Lifetime } \boldsymbol{\tau}(\mathbf{n s})\end{array}$ \\
\hline \hline & & & & & \\
0.000 & 4.315 & 0.004 & 0.908 & 0.996 & 0.920 \\
0.002 & 1.978 & 0.017 & 0.705 & 0.983 & 0.726 \\
0.004 & 1.688 & 0.056 & 0.605 & 0.944 & 0.666 \\
0.006 & 1.344 & 0.045 & 0.606 & 0.955 & 0.639 \\
0.008 & 1.413 & 0.024 & 0.596 & 0.976 & 0.615 \\
0.010 & 0.880 & 0.122 & 0.503 & 0.878 & 0.549 \\
\hline
\end{tabular}

Table 1: Fluorescence Lifetime Data of BPBD as a Function of Quencher Concentration in Toluene at Room Temperature.

The Stern-Volmer $(\mathrm{S}-\mathrm{V})$ plots $\mathrm{I}_{0} / \mathrm{I}\left(\right.$ or $\left.\tau_{0} / \tau\right)$ versus [Q] were plotted according to the equation [16],

$$
\frac{I_{0}}{I}\left(\operatorname{or} \frac{\tau_{0}}{\tau}\right)=1+K_{S V}[Q]
$$


where $\mathrm{K}_{\mathrm{SV}}$ is the $\mathrm{S}-\mathrm{V}$ quenching constant and [Q] is the quencher concentration. Eqn. (1) is applicable as long as the experimental results show linear variation. The S-V plot by steady state method in our case is found to be nonlinear showing positive deviation (Fig. 3). Whereas the S-V plot from time resolved method $\left(\tau_{0} / \tau\right.$ versus [Q]) is found to be linear (Fig. 4). It is observed that the phenomenon of quenching by time resolved method follows the S$\mathrm{V}$ relation. This suggests the presence of dynamic quenching. However, the positive deviation in the S-V plot from steady state measurements implies that the quenching is not purely dynamic and may be due to the presence of other parallel quenching mechanisms such as formation of either ground state complex, transient quenching process, static quenching process etc. [11].

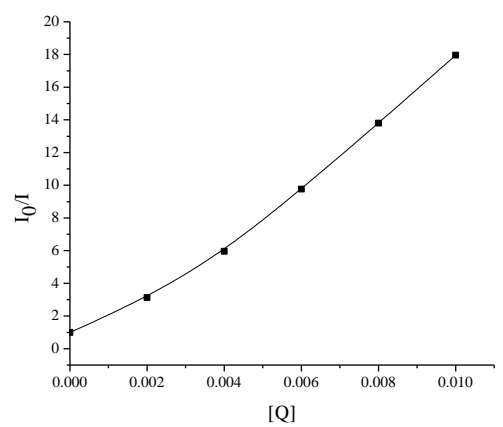

Fig. 3. S-V plot from steady state measurements

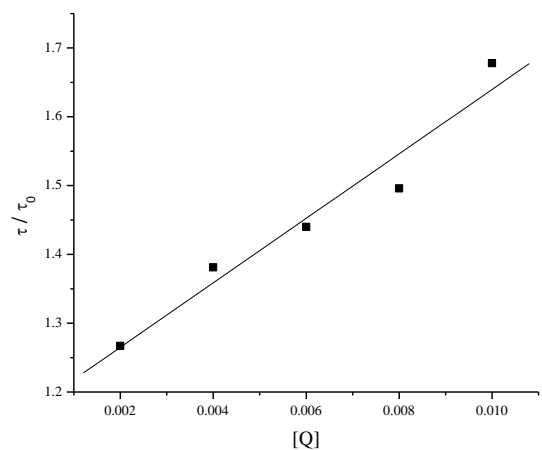

Fig. 4. S-V plot from transient measurements 


\subsection{Ground state complex formation model}

The possibility of ground-state complex formation has been analyzed by using the extended S-V equation given by the equation [3]

$$
\left[\left(I_{o} / I\right)-1\right] /[Q]=\left(K_{s v}+k_{g}\right)+\left(K_{s v} k_{g}\right)[Q]
$$

where $\mathrm{K}_{\mathrm{Sv}}$ and $\mathrm{kg}$ are $\mathrm{S}-\mathrm{V}$ and ground-state association constants respectively. According equation (3) the plot of $\left[\left(I_{0} / I\right)-1\right] /[Q]$ versus [Q] was found to be linear and is shown in Fig. 5(a). The correlation is good with correlation coefficient of 0.91 . From this plot, the values of $K_{\mathrm{sv}}$ and $k_{g}$ were determined. These values are tabulated in Table 2. Also, the value of $K_{\mathrm{sv}}$ was determined from the lower quencher concentration region of the plot $I_{0} / I$ versus $[Q]$ (Fig. $3)$. Corresponding values of $k_{q}$ were determined according to equation $k_{q}=K_{\mathrm{sv}} / \tau_{0}$. The value of $\tau_{0}$ was taken as $0.92 \mathrm{~ns}$ for BPBD in the absence of quencher. The value of $k_{q}$ determined from ground state complex formation model is represented as $k_{q}^{a}$ and one determined from lower concentration region of the plot $I_{0} / I$ versus [Q] is represented as $k_{q}^{b}$. From Table 2, it is observed that the values of $k_{\mathrm{q}}$ 's determined from two methods are not same. Therefore, fluorescence quenching due to ground state complex formation is ruled out in the present case. Also, we have observed that, the absorption spectrum of BPBD in toluene in the absence and presence of quencher do not show any observable difference in the shape or the peak position. This clearly indicates that the fluorescence quenching of BPBD has occurred without involving ground-state complex formation [11]. Alternatively, there may be transient component present in dynamic quenching. Transient fluorescence quenching occurs in those cases where quencher resides within a sphere of action with a volume surrounding the fluorophore at the time of relaxation. However, the presence of transient quenching is ruled out in the present case as we have observed a perfect linear plot for $\tau_{0} / \tau$ against [Q] (Fig. 4). Hence, the positive deviation could be due to the presence of static quenching. The possibility of static quenching process was analysed using sphere of action static quenching model $[1,2,17]$. 

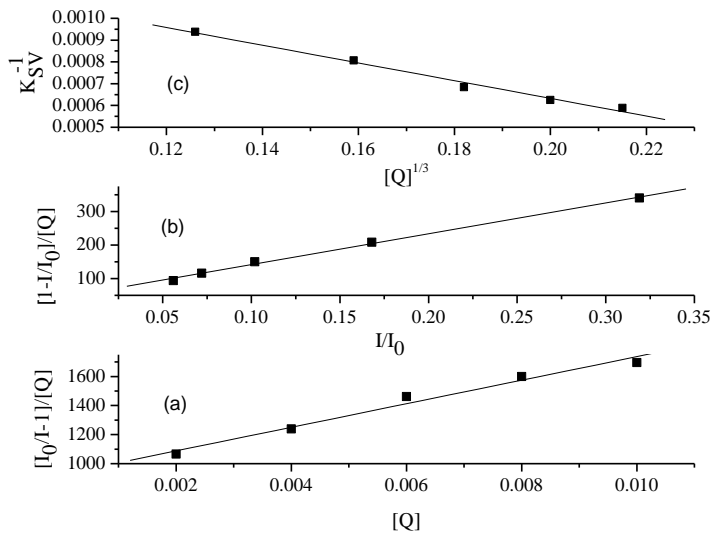

Fig. 5. Plots of (a) $[(\mathrm{I} 0 / \mathrm{I})-1] /[\mathrm{Q}]$ against $[\mathrm{Q}]$, (b) $[1-(\mathrm{I} / \mathrm{I} 0)] /[\mathrm{Q}]$ against $\mathrm{I} / \mathrm{I} 0$ and (c) Ksv-1 versus $[\mathrm{Q}] 1 / 3$

\begin{tabular}{|c|c|c|c|c|c|c|c|}
\hline $\begin{array}{c}k_{g} \\
\left(M^{-1}\right)\end{array}$ & $\begin{array}{c}k_{q}^{a} \times 10^{-10} \\
\left(M^{-1} s^{-1}\right)\end{array}$ & $\begin{array}{c}k_{q}^{b} \times 10^{-10} \\
\left(M^{-1} s^{-1}\right)\end{array}$ & $\begin{array}{c}K_{s v}^{c} \\
\left(M^{-1}\right)\end{array}$ & $\begin{array}{c}k_{q}^{c} \times 10^{10} \\
\left(M^{-1} s^{-1}\right)\end{array}$ & $\begin{array}{c}\text { Range of } \\
W\end{array}$ & $\begin{array}{c}\mathrm{V} \\
\left(M^{-1}\right)\end{array}$ & $\begin{array}{c}r \\
(\AA)\end{array}$ \\
\hline \hline 98.12 & 89.95 & 153.58 & 919.88 & 99.98 & $\begin{array}{c}0.50- \\
0.90\end{array}$ & 68.72 & 30.09 \\
& & 158 & & & \\
\hline
\end{tabular}

Table 2: The values Ksv, kg, kq, Range of W, V and R.

$$
R_{S}=4.31 \AA \quad R_{Q}=2.84 \AA \quad R\left(=R_{S}+R_{Q}\right)=7.15 \AA
$$

a Bimolecular quenching rate parameter determined from ground state complex formation model.

b Bimolecular quenching rate parameter determined from linear portion (low concentration) of the plots $I_{0} / I$ versus $[Q]$.

c Dynamic quenching constant and Bimolecular quenching rate parameters determined from sphere of action static quenching model.

\subsection{Sphere of action static quenching model}

According to this model, the instantaneous or static quenching occurs if the quencher molecule is very near to, or in contact with the fluorescent molecule at the exact moment it happens to be excited. This was explained by the fact that only a certain fraction ' $W$ ' of the excited state is actually quenched by the collisional mechanism. Some molecules in the excited state, the fraction of 
which is $(1-W)$ are de-activated almost instantaneously after being formed, because a quencher molecule happens to be randomly positioned in the proximity at the time the molecules are excited and interacts very strongly with them. Several models were employed to describe this static quenching process, all leading to the following modified form of the S-V equation [16].

$$
\left[1-\left(I / I_{o}\right)\right] / Q=K_{s v}\left(I / I_{o}\right)+(1-W) /[Q]
$$

According to equation (3), the plot of $\left[\left(1-I / I_{0}\right)\right] /[Q]$ against $I / I_{0}$ is found to be linear (Fig. 5(b)). The value of $\mathrm{K}_{\mathrm{sv}}$ is determined by least square fit method and hence $\mathrm{k}_{\mathrm{q}}$ is determined using the relation $\mathrm{k}_{\mathrm{q}}=\mathrm{K}_{\mathrm{sv}} / \tau_{0}$. These values are given in Table 2 . The intercept of least square fit line of Fig. 5(b) is equal to (1-W)/ [Q]. From this intercept, the static quenching constant ' $V$ ' and radii ' $r$ ' of sphere of action (or kinetic distance) is determined according to equations $\ln (1 / W)=V[Q]$ and $\mathrm{V} / \mathrm{N}^{\prime}=(4 / 3) \pi \mathrm{r}^{3}$, where $N^{\prime}$ is Avogadro's number per millimole. The respective data is collected in Table 2. It is observed that the value of $\mathrm{K}_{\mathrm{SV}}$ is rather larger relative to the value of $\mathrm{V}$, which explains the lack of the changes in absorption spectrum in the presence of quencher. In order to compare the radius ' $r$ ' of sphere of action with encounter distance or reactive distance $R$, i.e., the sum of the radii of the BPBD ( $\left.R_{S}\right)$ and the quencher molecule $\left(R_{Q}\right)$, the radii of BPBD and aniline were determined by the method suggested by Edward [18] and are given at the bottom of Table 2. It is known that the static effect takes place in case of steady state experiments irrespective of ground state complex formation, when the distance between the quencher molecule and the excited molecule lies between the reactive distance $\mathrm{R}$ and kinetic distance $\mathrm{r}$, provided the reactions are diffusion limited [19, 20]. From Table 2 it is evident that, the value of kinetic distance ' $r$ ' is greater than the reactive distance $R$, indicating the role of static quenching process in the present case. Further, to find out whether the reaction is diffusion limited, we have used the finite sink approximation model.

\subsection{Finite sink approximation model}

According to this model, the modified S-V equation is [21] 


$$
K_{s v}^{-1}=\left(K_{s v}^{o}\right)^{-1}-\frac{\left(2 \pi N^{\prime}\right)^{1 / 3}}{4 \pi N^{\prime} D \tau}[Q]^{1 / 3}
$$

where $K_{s v}^{o}$ is the $\mathrm{S}-\mathrm{V}$ constant at [Q] $=0$ and is given by $K_{s v}^{o}=\frac{4 \pi N^{\prime} D R \tau k_{a}}{4 \pi N^{\prime} D R+k_{a}}, \mathrm{D}$ is mutual diffusion coefficient, $\mathrm{k}_{\mathrm{a}}$ is activation energy controlled rate constant and other terms have their usual meanings. According to equation (5), the plot of $K_{s v}^{-1}$ against $[Q]^{1 / 3}$ which is shown in Fig. 5(c) is found to be linear with negative slope. The value of $\mathrm{D}$ is calculated from the slope and the distance parameter $R^{\prime}$ is calculated from the intercept according to equation $K_{s v}^{0}=4 \pi N^{\prime} D R^{\prime} \tau$. These values are given in Table 3 . For $R^{\prime}>\mathrm{R}$, the bimolecular reactions of fluorescence quenching are said to be diffusion limited, if the value of $\mathrm{k}_{\mathrm{q}}\left(k_{q}^{c}\right)$ determined from Eqn. (3) is greater than $4 \pi N^{\prime} R^{\prime} D$ [20, 22]. From the Tables $2 \& 3$, it is observed that $k_{q}^{c}>4 \pi N^{\prime} R^{\prime} D$. Therefore, the reaction is diffusion limited.

\begin{tabular}{|c|c|c|c|}
\hline $\begin{array}{c}K_{s v}^{o} \\
\left(M^{-1}\right)\end{array}$ & $\begin{array}{c}D \times 10^{5} \\
\left(\mathrm{~cm}^{2} s^{-1}\right)\end{array}$ & $\begin{array}{c}R^{\prime} \\
(\AA)\end{array}$ & $\begin{array}{c}4 \Pi N^{\prime} R^{\prime} D \times 10^{-10} \\
\left(M^{-1} s^{-1}\right)\end{array}$ \\
\hline \hline 689.65 & 55.05 & 17.98 & 74.93 \\
\hline
\end{tabular}

Table 3: The values of $K_{s v}^{o}$, Mutual Diffusion Coefficient D, Distance Parameter R' and $4 \pi \mathrm{N}^{\prime} \mathrm{DR}$ '.

\section{Conclusion}

From the present investigation we conclude that: (1) the S-V plot shows positive deviation leading to high value of $\mathrm{kq}$, indicating efficient fluorescence quenching. (2) Ground state complex formation is not playing a role in quenching mechanism. (3) The linear S-V plot from time resolved method indicates the absence of transient fluorescence quenching. (4) The value of kinetic distance ' $r$ ' is greater than the encounter distance $R$, suggesting the role of static quenching (5) The value of $k_{q}$ is greater than $4 \pi N^{\prime} R^{\prime} D$, 
indicating the biomolecular reaction is diffusion limited. In view of the above facts we conclude that fluorescence quenching of BPBD by aniline in toluene is due to simultaneous presence of dynamic and static quenching processes.

\section{Acknowledgement}

The authors are grateful to Dr H Pal, Chemical Dynamics Division, BARC, Mumbai for providing TCSPC facility and Dr S Kumar B Hadimani for providing the necessary scintillation sample used in the present work.

\section{References}

[1] H. R. Deepa, J. Thipperudrappa and H. M. Suresh Kumar, "Fluorescence quenching studies of 6,7,8,9-tetrahydro-6,8,9trimethyl-4-(trifluoromethyl)-2H-pyrano[2,3-b][1,8]naphthyridin2-one by aromatic amines in alcohols," Int. J. Phy. \& Appl., vol. 4(2), p. 157, 2012. ISSN 0974-3103.

[2] H. R. Deepa, J. Thipperudrappa, and H. M. Suresh Kumar, " A study on fluorescence quenching of LD-425 by aromatic amines in 1,4-dioxane-acetonitrile mixtures," J. Lumin., vol. 132, p. 1382, 2012. http://dx.doi.org/10.1016/j.jlumin.2012.01.008.

[3] B. G. Evale, and S. M. Hanagodimath, "Static and dynamic quenching of biologically active coumarin derivative by aniline in benzene-acetonitrile mixtures," J. Lumin., vol. 130, p. 1330, 2010. http://dx.doi.org/10.1016/j.jlumin.2010.03.011.

[4] Y. Ni, S. Su, and S. Kokot, "Spectrometric studies on the interaction of fluoroquinolones and bovine serum albumin," Spectrochim. Acta Part A, vol. 75, p. 547, 2010. http://dx.doi.org/10.1016/j.saa.2009.11.014.

[5] B. G. Evale, and S. M. Hanagodimath, "Effect of temperature and quencher on the fluorescence of 4-(5-methyl-3-furan-2-ylbenzofuran-2-yl)-7-methyl-chromen-2-one in different solvents" Spectrochim. Acta Part A, vol. 75, p. 1592, 2010. http://dx.doi.org/10.1016/j.saa.2010.02.024.

[6] S. M. Hanagodimath, B. Siddlingeshwar, J. Thipperudrappa, S. K. B. Hadimani, "Fluorescence-quenching studies and temperature dependence of fluorescence quantum yield, decay time and intersystem crossing activation energy of TPB" J. Lumin., vol. 129, p.335, 2009. http://dx.doi.org/10.1016/j.jlumin.2008.10.016. 
[7] R. M. Melavanki, R. A. Kusanur, J. S. Kadadevaramath, and M. V. Kulakarni, "Quenching mechanisms of 5BAMC by aniline in different solvents using Stern-Volmer plots", J. Lumin., vol. 129, p.1298, 2009. http://dx.doi.org/10.1016/j.jlumin.2009.06.011.

[8] C. Tablet and M. Hillerbrand, "Quenching of fluorescence of 3carboxy-5,6-benzocoumarin by aromatic amines", J. Photochem. Photobiol. A Chem., vol. 189, p. 73, 2007. http://dx.doi.org/ 10.1016/j.jphotochem.2007.01.009.

[9] D. S. Biradar, J. Thipperudrappa and S.M. Hanagodimath, "Fluorescence quenching studies of 1, 3 - diphenyl benzene,", Spectrosc. Lett., vol. 40, p. 559, 2007. http://dx.doi.org/ 10.1080/00387010701301147.

[10] C. Hariharan, A. K. Mishra, "Quenching of liquid scintillators by chloroalkanes and chloroalkenes", Radiat. Measure. vol. 32, p. 113, 2000. http:/ / dx.doi.org/10.1016/S1350-4487 (99)00246-2.

[11]P. K. Behera and A. K. Mishra, "Static and dynamic model for 1napthol fluorescence quenching by $\mathrm{CCl}_{4}$ in dioxane-acetonitrile mixtures", J. Photochem. Photobiol. Chem. Part A, vol. 71, p. 115, 1993. http://dx.doi.org/10.1016/1010-6030(93)85061-C.

[12] G. Santos, F. J. Fonseca, A. M. Andrade, I. Grova, L. Akcelrud, L. Pereira, "Polymer light emitting diode using PFT--poly(9,9'-ndihexil-2,7-fluorenodiilvinylene-alt-2,5thiophene, J.Nanosci. Nanotechnol., vol. 10, p. 2776, 2010. http://dx.doi.org /10.1166/ jnn.2010.1410.

[13] B. Debasis, Q. Lei, T. Teng-Kuan,H. H. Paul, "Quantum Dots and Their Multimodal Applications: A Review", Material., vol. 3, p. 2260, 2010. http:/ / dx. doi.org/10.3390/ma3042260.

[14] M. Takiue, and H. Ishikawa, "Improvement of Cherenkov counting method (author's transl)", Radioisotopes., vol. 27, p. 123127, 1978. ISSN:0033-8303.

[15] G. Gezelius, "A convenient, efficient and inexpensive method for scintillation counting of polyacrylamide gel slices after electrophoresis", Analy. Biochem, vol. 80, p. 627, 1977. http:/ /dx.doi.org/10.1016/0003-2697(77)90688-1.

[16] J. R. Lakowicz, Principles of Fluorescence Spectroscopy, $3^{\text {rd }}$ Ed., Springer, New York, 2006.

[17] M. N. Swaminathan, and N. Radha," Static and dynamic quenching model for 4-amino diphenyl fluorescence quenching by CCl4 in hexane", Spectrochim. Acta Part A, vol. 60, p.1839, 2004. http:/ /dx.doi.org/10.1016/j.saa.2003.09.023. 
[18] J. T. Edward, "Molecular volumes and the Stokes-Einstein equation", J. Chem. Edu., vol. 47, p. 261, 1970. http://dx.doi.org /10.1021/ed047p261.

[19] J. C. Andre, M. Niclause, and W.R. Ware, "Kinetics of partly diffusion controlled reactions I. Transient and apparent transient effect in fluorescence quenching", Chem. Phys., vol. 28, p. 371, 1978. http://dx.doi.org/10.1016/0301-0104 (78)80014-7.

[20] H. Zeng and G. Durocher, "Analysis of fluorescence quenching in some antioxidants from nonlinear Stern-Volmer Plots", J. Lumin., vol. 63, pp. 74-84, 1995. http://dx.doi.org/10.1016/00222313(94)00045-E.

[21] J. Keizer, "Diffusion effects on rapid bimolecular reactions", Chem. Rev., vol. 87, p. 167, 1987. http://dx.doi.org /10.1021/ cr00077a009.

[22] G. C. Joshi, R. Bhatnagar, S. Doraiswamy, and N. Periaswamy, "Diffusion controlled reactions: Transient effects in the fluorescence quenching of indole and $\mathrm{N}$-acetyltryptophanamide in water", J. Phys. Chem., vol. 94, p. 2908, 1990. http://dx.doi.org /10.1021/j100370a033. 